\title{
Metal-organics: A rich seam of data for knowledge mining
}

\section{Peter A. Wood ${ }^{1}$}

${ }^{1}$ The Cambridge Crystallographic Data Centre, 12 Union Road, Cambridge, CB2 1EZ, UK.

E-mail:wood@ccdc.cam.ac.uk

In the last year, the Cambridge Structural Database (CSD) has grown to encompass over 875,000 entries and it could potentially reach a million entries before the next ACA meeting in 2018! As the number of entries has increased in recent decades, the fraction of metal-organic entries has steadily increased, accounting for more than half of the CSD since 1991 and reaching 57\% of all entries at the start of 2017. Despite this abundance of structural data on metal-organic systems, there is still relatively little research in detailed structural analysis, design and prediction of metal-organic compounds compared to the purely organic field. We think this area has a lot of potential for effective use of knowledge-mining concepts and applications.

Here we will present some of the improvements currently being made to the CSD software to aid searching, visualisation and analysis of metal-organic structures as well as ideas for what to do next. This is also a partially crowd-sourced poster as we will be encouraging you, the ACA community, to give your thoughts on what else we should improve and what the highest priority should be! 